\title{
Adherence to maintenance medication in asthma in patients admitted with acute asthma
}

\begin{abstract}
Introduction: International guidelines for asthma treatment are described by GINA. The regular use of inhaled corticosteroid (ICS) is the recommended and most effective treatment of persistent asthma. However, suboptimal adherence to ICS maintenance treatment is a well-known occurrence among asthmatics leading to uncontrolled asthma and poor outcomes.
\end{abstract}

Objectives: Assess the proportion of no adherence in patients admitted with acute asthma and identify determinants that potentially influence patients' adherence to ICS treatment.

Methods: Performed as a retrospective descriptive study, by retrieving data from a cohort study at the Acute Medical Department (AMA) at Odense University Hospital (OUH), we identified 191 patients admitted with acute asthma between 1/8-200931/11-2011. Adherence to ICS was calculated and patients medicinal use history was obtained by electronic prescription database.

Results: Non adherence to ICS was found in $61.3 \%$ of the total population of which $36.1 \%$ were completely non-users of ICS. Women comprised $64.4 \%$ of study population and the median age was 38years. Distribution of patients that were adherent to ICS was found to increase with older age and higher annual use of reliever medication. Adherence was found in $61.9 \%$ and $65.6 \%$ of patients who had dispensed oral corticosteroids (OCS) and those who used fixed dose combinations (FDC) of ICS and long-acting 32 -agonists (LABA), respectively. While a larger proportion of patients who used antidepressant/antipsychotic and those who had no co morbid conditions were found to be non adherent to ICS.

Conclusion: As a large proportion of patients admitted with acute asthma attacks were non-users of ICS. Future actions should focus on implementation of guidelines.

Keywords: asthma, adherence, inhaled corticosteroids, exacerbation, maintenance treatment, hospitalized
Volume I Issue I - 2014

\author{
Tho Chi Nguyen,' Hanne Madsen,' Anton \\ Pottegard, ${ }^{2}$ Annmarie Lassen ${ }^{2}$ \\ 'Department of Respiratory Medicine, Odense University \\ Hospital, Denmark \\ ${ }^{2}$ Department of Clinical Pharmacology, University of Southern \\ Denmark, Denmark
}

Correspondence: Hanne Madsen, Department of Respiratory Medicine, Odense University Hospital, Sdr. Boulevard 29, 5000 Odense C, Denmark, Tel 4058004I, Email h.madsen@rsyd.dk

Received: August 09, 2014 | Published: August 21, 2014
Abbreviations: AMA, acute medical department; ATC, anatomical therapeutic chemical; COPD, chronic obstructive pulmonary disease; CPR, central person register; CRP, C-reactive protein; DDD, defined daily doses; EPJ, electronic patients journal; FDC, fixed dose combination; FPAS, funen patients administrative system; GINA, global initiative for asthma; ICD-10, international classification of disease $-10^{\text {th }}$ edition; ICS, inhaled corticosteroids; ICU, intensive care unit; LABA, long-acting beta-2-agonists; OCS, oral corticosteroids; OPED, odense pharmacoepidemiologic database; OUH, odense university hospital; SABA, short-acting beta-2-agonists; WHO, world health organization

\section{Introduction}

Asthma is a chronic inflammatory disorder of the airways with symptoms such as recurrent episodes of breathlessness, coughing, wheezing and chest tightness. ${ }^{1}$ Prevalence for asthma in Denmark is estimated to be $6.4 \%$ within the adult population. ${ }^{2}$ The majority of these suffer from mild to moderate asthma that can be adequately treated with regular use of inhaled corticosteroids (ICS), in addition to short-acting $\beta 2$-agonists (SABA) as reliever medication. Meanwhile approximately $5 \%$ are thought to suffer from "difficult asthma" which despite treatment with high-dose therapy of ICS combined with the use of long-acting $\beta 2$-agonist (LABA), still has persistent symptoms and/or frequent exacerbations. ${ }^{3}$ Suboptimal adherence to ICS treatment is likely to be the reason for difficult asthma and is a well-known problematic among asthmatics leading to poor asthma control, reduced quality of life, decreasing lung function, increasing frequency of hospitalization and asthma mortality. ${ }^{4-7}$ This subgroup are also likely responsible for a large proportion of the annual healthcare cost of asthmatics because of their more frequent visits to emergency department and hospitalization. ${ }^{3,8,9}$ Rates of non-adherence is reported in a 1997 review to range between 30\% and 70\% among asthmatics, this include all asthma medications and is irrespective of how adherence is measured. ${ }^{10}$

International guidelines for treatment are described by Global Initiative for Asthma (GINA). Treatments are dependent on severity of the disease and adjusted by level of symptom control are described in 5 steps with increasing efficacy. Step 1 is reserved for patients with mild asthma, an as needed reliever medication (i.e. rapid-acting $\beta 2$-agonist) can be used only. Where asin step 2-5, regular maintenance therapy with ICS in addition to as needed reliever medication is recommended for all patients with persistent asthma. With the addition of LABA for patients who remain symptomatic on an ICS alone. ${ }^{1}$

Many factors are associated with adherence to maintenance 
treatment of asthma, hospitalization and emergency visits. The use of SABA was suggested to be preferred over maintenance treatment among patients with uncontrolled asthma. ${ }^{11}$ This could lead to under use of control therapy resulting in hospitalization. On the other hand, fixed dose combination (FDC) of LABA with ICS has been shown to increase the use of regular ICS among asthmatics since it have been introduced in Denmark. ${ }^{12}$ Age, gender, use of oral glucocorticosteroids (OCS), antidepressants/antipsychotics has all been suggested as factors influencing adherence to ICS treatment.

Our aim in this study was to assess the rate of non adherence to ICS among patients hospitalized for acute exacerbation, and to identify patients' characteristics or other factors that potentially influence adherence to ICS controller therapy among asthmatics.

\section{Materials and methods}

\section{Settings and design}

This study was performed as a descriptive retrospective analysis on adult patients (i.e. $\geq 15$ years) admitted due to asthma exacerbation to Odense University Hospital (OUH) in the county of Funen, Denmark. Patients were retrieved from an earlier conducted cohort study at the Acute Medical Department (AMA) at OUH which existed between 2008-2011, and by using a prescription database, Odense Pharmacoepidemiologic Database (OPED) their history of medical use were obtained.

\section{Data sources}

OPED is a research database based on information on reimbursed drug dispensed over the counter, and has since October 1990 covered all pharmacies in the county of Funen ${ }^{13}$ Each prescription record contains a unique person identifier, age and gender, date of purchase, brand name, active substance, quantity, and form of the drug. The substances are registered according to WHO's Anatomical Therapeutic Chemical (ATC) code and quantities measured in defined daily doses (DDD) methodology. ${ }^{14}$ The indication for treatment and dosing instruction are not recorded. Patients were identified using Funen Patient Administrative System (FPAS) by their CPR-number (i.e. social security number) and indication using the ICD-10 code.

\section{Study population}

When admitted to OUH, patients first arrive at the AMA were vitals are being measured and registered to the Electronic Patients Journal (EPJ)by indication using ICD-10 code, and later on discharged from AMA to be transferred to other departments. From these discharge summaries obtained from FPAS the AMA-cohort study obtained patient information which we subsequently retrieved data from. All patients who were admitted to OUH in the period of 1/8-2009-31/112011 with asthma as main diagnosis ICD-10, J45 (asthma) or J46 (status asthmaticus) were included in this study. Patients arriving at the AMA with a severe critical status would immediately be transferred to the intensive care unit (ICU); data from these patients would not be obtained by AMA-cohort and therefore, those patients were not included in this study. Same goes for children under the age of 15 which would be transferred directly to paediatric department.

\section{Data analysis and drug use variables}

All information on subjects' medical use history in the 12 months before admission was extracted using OPED. Our main outcome was adherence to controller medication, mainly ICS use prior to hospitalisation. We compared the distribution of patients that were adherent and nonadherent to ICS to patients' characteristics and history of medical use to identify possible determinates for adherence to ICS therapy. Patient demographics and other covariates considered were gender, age, comorbid conditions and patients C-reactive protein (CRP) levels to detect possible respiratory infection. We stratified subjects into four age categories, $\geq 15$-29years, 30-44years, $45-64$ years and $\geq 65$ years. Admission arterial blood gas value such as $\mathrm{pH}, \mathrm{pO}_{2}, \mathrm{pCO}_{2}$ and duration of admission was recorded to determine severity of condition.

Anti-asthmatic drugs considered as factors associated with ICS use was annual SABA consumption, or whether combination with LABA were used, or ICS as mono therapy, as well as oral corticosteroids (OCS) as rescue treatment. We categorised subjects according to their annual SABA consumption in DDD (1-99, 100-199, 200-399 and $\geq 400 \mathrm{DDD} /$ year). SABA is referred to as inhaled short-acting beta2-agonists with active substance salbutamol andterbutaline (ATC R03AC02 and R03AC03). Patient use of a particular drug was defined as at least one redeemed prescription during the year preceding the admission date. Other drugs included were anticholinergic (R03BB), oral glucocorticosteroids (ATC H02AB), systemic antibiotics use for treatment of possible respiratory infection (ATC J01CE02, J01CA01, J01CA02，J01CA04，J01FA01，J01CR02，J01FA06，J01FA09 and J01FA10), antipsychotics (ATC N05A) and antidepressants (ATC N06A).

Because records of dosing instructions were missing, to define adherence we used the DDD as a surrogate method of measurement. The DDD represents the assumed average maintenance dose per day for a drug used for its main indication in adults. It should be emphasised that the DDD is a unit of measurement and should not be mistaken for the recommended or the prescribed daily doses. ${ }^{14}$ DDDs for the most frequently used anti-asthmatic drugs in our materials are presented in Table 1. While dosing regimen vary between patients depending on severity of disease, according to our on board physician as well as earlier long term study on ICS use suggest that a low dose of ICS might be sufficient to maintain asthmatic control as long as it is being used on a regular basis. ${ }^{6}$ In addition we based our numbers on the lowest recommended daily dose of $200 \mu \mathrm{g}$ of budesonide as maintenance treatment for asthma. ${ }^{1,15}$ Based on these considerations, adherence to ICS was set as $>25 \%$ of DDD for the given active substance and was measured by the cumulative dispensed ICS in the 12 months preceding admission date converted to DDD. To exclude any overestimates of excess dispensed medicine due to worsening of symptoms, ICS filled in the period of 2 weeks prior to admission date was not included. To account for canisters filled just before the observation time, regardless of quantity, canister were assigned a duration of 90days and the proportion of a prescription that overlapped with 12 month observation window was added. The same corrections were made with canisters filled within the last 90days before the admission. All patients that have dispensed an annual ICS doses equivalent to $>25 \%$ of the calculated annual DDD was in the adherence-group, patients with lower annual ICS dispensed were nonadherence to ICS. ICS referred to inhaled corticosteroid in non-combination inhalers with active substance beclometasone, budesonide and fluticasone (ATC R03BA01, R03BA02 and R03BA05) respectively, or fixed dose combination (FDC) with ICS and LABA (ATC R03AK06 and R03AK07).

Registry-based studies do not require an ethical approval in Denmark. The Danish Data Protection Agency gave permission to data Access. 
Table I Defined daily doses (DDD) for the most frequently used anti-asthmatics in Funen County, Denmark. ${ }^{14}$

\begin{tabular}{|c|c|c|c|c|}
\hline ATC-code & Drug & DDD & Unit & \\
\hline \multirow[t]{3}{*}{ R03BA0I } & Beclometasone & 0.8 & $\mathrm{mg}$ & Inhal.aerosol \\
\hline & & 0.8 & $\mathrm{mg}$ & Inhal.powder \\
\hline & & 1.5 & $\mathrm{mg}$ & Inhal.solution \\
\hline \multirow[t]{3}{*}{ R03BA02 } & Budesonide & 0.8 & $\mathrm{mg}$ & Inhal.aerosol \\
\hline & & 0.8 & $\mathrm{mg}$ & Inhal.powder \\
\hline & & 1.5 & $\mathrm{mg}$ & Inhal.solution \\
\hline \multirow[t]{3}{*}{ R03BA05 } & Fluticasone & 0.6 & $\mathrm{mg}$ & Inhal.aerosol \\
\hline & & 0.6 & $\mathrm{mg}$ & Inhal.powder \\
\hline & & 1.5 & $\mathrm{mg}$ & Inhal.solution \\
\hline \multirow[t]{3}{*}{ R03AC02 } & Salbutamol & 0.8 & $\mathrm{mg}$ & Inhal.aerosol \\
\hline & & 0.8 & $\mathrm{mg}$ & Inhal.powder \\
\hline & & 10 & $\mathrm{mg}$ & Inhal.solution \\
\hline \multirow[t]{3}{*}{ R03AC03 } & Terbutaline & 2 & $\mathrm{mg}$ & Inhal.aerosol \\
\hline & & 2 & $\mathrm{mg}$ & Inhal.powder \\
\hline & & 20 & $\mathrm{mg}$ & Inhal.solution \\
\hline \multirow[t]{2}{*}{ R03ACI 2} & Salmeterol & 0.1 & $\mathrm{mg}$ & Inhal.aerosol \\
\hline & & 0.1 & $\mathrm{mg}$ & Inhal.powder \\
\hline \multirow[t]{2}{*}{ R03ACI 3} & Formoterol & 24 & mcg & Inhal.aerosol \\
\hline & & 24 & $\mathrm{mcg}$ & Inhal.powder \\
\hline
\end{tabular}

\section{Results}

A total 191 admissions to OUH were identified with the main diagnosis of asthma between 2009 and 2011. Patient demographics and clinical data are presented in Table 2. Women comprised $64.4 \%$ of the total number of subject. Results showed no differences on level of adherence to ICS between genders. Median age was found to be higher (47years) among patients adherent to ICS compared to the nonadherent-group (34years). The majority of patients were found in the youngest age categories 15-29years and 30-44years, comprised of 124 patients whereas $70.1 \%$ of these were nonadherent to ICS. In contrast $63.6 \%$ of patients $\geq 65$ years was adherent to ICS treatment. The gradual increase in adherence level by age categories found in this study is shown in Figure 1.

At admission, possible upper respiratory infections were reported in $23.0 \%$ of the patients, determined by CRP-levels. Comorbidity by other conditions in this study population were low, with $76.4 \%$ of patients with Charlson ${ }^{16}$ comorbidity index score $=0$ or 1 . Of those with comorbidity-score $\geq 2$ no difference in adherence level were found. Only in patients with comorbidity score $=0,47$ of 62 patients
(75.8\%) were found to be nonadherent to ICS. Between groups comparison of arterial blood gas measurement showed near identical results for adherent and nonadherent patients for $\mathrm{pH}, \mathrm{pO}_{2}$ and $\mathrm{pCO}_{2}$ values (Table 2), as well as days of admission.

Patient's medical use history is presented in Table 3. Pharmacy records showed that nonadherence to ICS occurred in $61.3 \%$ of patients (117 of 191). Of these, 69 patients were found to not use any ICS the year before they were admitted, comprising of $36.1 \%$ of the study population. Among ICS users, 96 patients (78.7\%) were treated with FDC of ISC/LABA, where as the majority of these were adherent to ICS $(65.6 \%)$. There was a trend towards increasing annual SABA use and the higher rate of adherence to ICS therapy found in this study (Figure 2). 132 of the 191(69.1\%) patients had an annual use of SABA $\leq 99 \mathrm{DDD} /$ year, which correspond to 2 or less daily dose of reliever therapy per week the year preceding admission, which of $67.4 \%(n=89)$ were nonadherent to ICS treatment.

Rescue treatment (i.e. OCS) and antibiotics were dispensed to $33.0 \%$ and $50.3 \%$, respectively, of the overall population in the year prior to admissions. Of these, a median of 2 fillings on both OCS and antibiotics were dispensed, where as adherence were found in the larger proportion of those used OCS $(n=39,61.9 \%)$. Only 11 patients have dispensed antipsychotics prior to admission date, whereas 8 of these were nonadherent to ICS. Among the 32 patients using anti depressants, nonadherent to ICS treatment were found in $75 \%$ of these (Table 3).

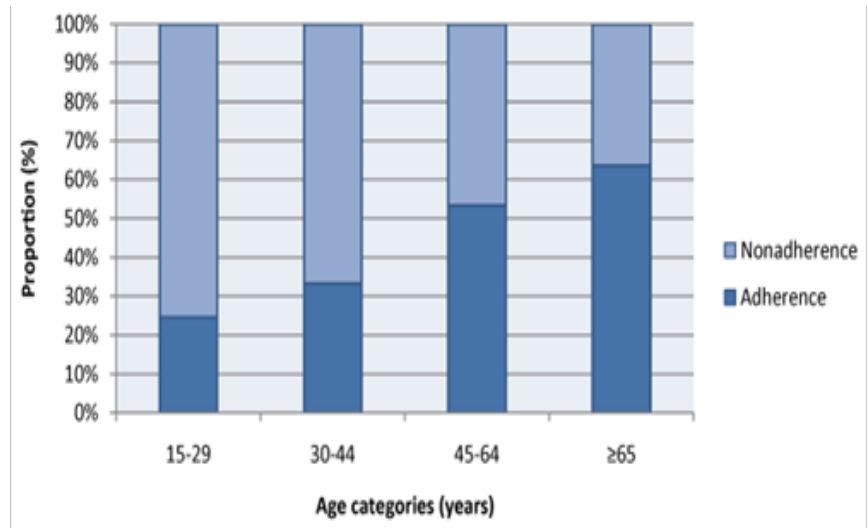

Figure I Proportion of patients adherent and nonadherent to ICS in increasing age categories.

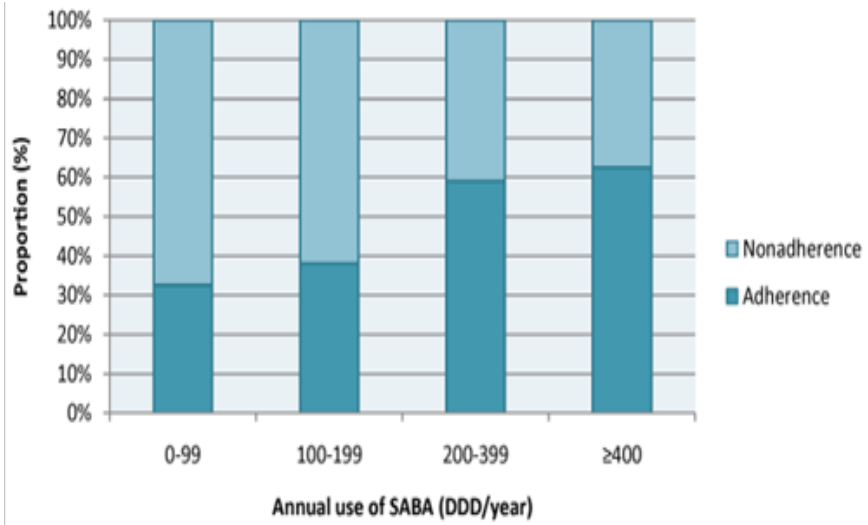

Figure 2 Proportion of patients adherent and nonadherent to ICS in increasing annual SABA use categories. 
Table 2 Baseline characteristics, demographics and clinical data

\begin{tabular}{|c|c|c|c|}
\hline & All Patients $(N=191)$ & Adherence $(n=74)$ & Nonadherence $(n=|| 7)$ \\
\hline \multicolumn{4}{|l|}{ Gender, n (\%) } \\
\hline Male & $68(35.6 \%)$ & $25(33.8 \%)$ & $43(36.8 \%)$ \\
\hline Female & $123(64.4 \%)$ & $49(66.2 \%)$ & $74(63.2 \%)$ \\
\hline Age, median(IQR)(years) & $38(25-54)$ & $47(32-62)$ & $34(22-44)$ \\
\hline \multicolumn{4}{|l|}{ Age groups, n (\%) } \\
\hline $15-29$ & $6 \mathrm{I}(3 \mathrm{I} .9 \%)$ & $15(20.3 \%)$ & $46(39.3 \%)$ \\
\hline $30-44$ & $63(33.0 \%)$ & $21(28.4 \%)$ & $42(35.9 \%)$ \\
\hline $45-64$ & $45(23.6 \%)$ & $24(32.4 \%)$ & $21(17.9 \%)$ \\
\hline 65 & $22(11.5 \%)$ & $14(18.9 \%)$ & $8(6.8 \%)$ \\
\hline \multicolumn{4}{|l|}{ Charlsoncomorbidity index, n (\%) } \\
\hline 0 & $62(32.5 \%)$ & $15(20.3 \%)$ & $47(40.2 \%)$ \\
\hline I & $94(49.2 \%)$ & $44(59.5 \%)$ & $50(42.7 \%)$ \\
\hline$\geq 2$ & $35(18.3 \%)$ & $15(20.3 \%)$ & $20(17.1 \%)$ \\
\hline \multicolumn{4}{|l|}{ Arterial blood gas, mean $\pm S D$} \\
\hline $\mathrm{pH}$ & $7.44 \pm 0.07$ & $7.45 \pm 0.07$ & $7.44 \pm 0.07$ \\
\hline $\mathrm{pO}_{2}$ & $11.04 \pm 3.87$ & $1 \mathrm{I} .14 \pm 3.40$ & $\mid 0.97 \pm 4.21$ \\
\hline $\mathrm{PCO}_{2}$ & $4.62 \pm 1.14$ & $4.64 \pm 0.93$ & $4.6 I \pm I .28$ \\
\hline CRP & $16.56 \pm 23.40$ & $14.80 \pm 22.88$ & $17.68 \pm 23.77$ \\
\hline CRP >60, n (\%) & $44(23.0 \%)$ & $17(23.0 \%)$ & $27(23.1 \%)$ \\
\hline Duration of admission, median(IQR)(days) & $2(2-3)$ & $2(2-4)$ & $2(1-3)$ \\
\hline
\end{tabular}

IQR, inter quartile range; $S D$, standard deviation; $N$, number of patients total; $n$, number of patients subgroup

Table 3 Patients history of drug use the year prior to admission

\begin{tabular}{|c|c|c|c|}
\hline Drug use, n (\%) & All Patients $(N=\mid 91)$ & Adherence $(n=74)$ & Nonadherence $(n=|| 7)$ \\
\hline \multicolumn{4}{|l|}{ Use of ICS } \\
\hline Mono therapy & $26(\mid 3.6 \%)$ & II (I4.9\%) & $15(12.8 \%)$ \\
\hline Combination with LABA & $96(50.3 \%)$ & $63(85.1 \%)$ & $33(28.2 \%)$ \\
\hline No use of ICS & $69(36.1 \%)$ & $0(0.0 \%)$ & $69(59.0 \%)$ \\
\hline \multicolumn{4}{|l|}{ Use of SABA(DDD/year) } \\
\hline $0-99$ & $132(69.1 \%)$ & $43(58.1 \%)$ & $89(76.1 \%)$ \\
\hline $100-199$ & $21(11.0 \%)$ & $8(10.8 \%)$ & $13(11.1 \%)$ \\
\hline 200-399 & $22(11.5 \%)$ & $13(17.6 \%)$ & $9(7.7 \%)$ \\
\hline$\geq 400$ & $16(8.4 \%)$ & $10(13.5 \%)$ & $6(5.1 \%)$ \\
\hline \multicolumn{4}{|l|}{ Use of other drugs(yes/no) } \\
\hline Oral corticosteroid & $63(33.0 \%)$ & $39(52.7 \%)$ & $24(20.5 \%)$ \\
\hline Antibiotics & $97(50.8 \%)$ & $46(62.2 \%)$ & $51(43.6 \%)$ \\
\hline Antipsychotics & II (5.8\%) & $3(4.1 \%)$ & $8(6.8 \%)$ \\
\hline Antidepressants & $32(16.8 \%)$ & $8(10.8 \%)$ & $24(20.5 \%)$ \\
\hline Anticholinergic(inhaled) & $20(10.5 \%)$ & $12(16.2 \%)$ & $8(6.8 \%)$ \\
\hline
\end{tabular}

ICS, inhaled corticosteroids; LABA, long acting beta-2-agonist; SABA, short acting beta-2-agonists; DDD, defined daily doses; $\mathrm{N}$, number of patients total; $\mathrm{n}$, number of patients subgroup

Results are presented as number of patient n, and percent(\%) 


\section{Discussion}

In between-group comparison, this study demonstrates that a larger proportion of patients hospitalized by asthma were poorly adherent to ICS therapy. Which coincide with others findings that nonadherence to regular ICS therapy is associated with uncontrolled asthma and increasing rate of hospitalization. ${ }^{4,6,8}$ The fact that one third of our study population did not use any ICS as maintenance treatment was surprising, considering that patients hospitalized with acute asthma in fact are those in need of regular ICS treatment. The lack of verified asthma diagnosis meant we could not distinguish whether non-use of ICS was due to primary nonadherence (i.e. non-fillings), or that patients were diagnosed as having milder asthma (GINA step 1) and/or as first time admission of asthma and therefore not prescribed control therapy prior to hospitalisation. Williams et al., ${ }^{17}$ found the rate of primary nonadherence to ICS among asthmatics to $8 \%$, although other studies suggested the rate of non filling to be much higher at 30\%. ${ }^{18,19}$ By these rates of primary nonadherence, excluding non-users of ICS would underestimate the rate of non adherence in our findings.

Since 2000, FDC with ICS and LABA combined in one inhaler were introduced and extensively marketed in Denmark. Since then prevalence of ICS use among young adult's asthmatics has increased. ${ }^{12}$ The use of FDC have been found to improve lung function, asthma control and exacerbation rate compared to separate use of ICS and LABA or mono therapy with ICS. ${ }^{20-22}$ We found in this study that twice as many patients in use of FDC were adherent to treatment to those who were in treatment with ICS alone. At first, this seems to indicate that the use of FDC improved rate of adherence. However, results are contradictory as our study comprised only of admitted patients, which is an indication for uncontrolled asthma.

Age-related adherence (Figure 1) to controller therapy might be explained by the subjective perception of the "need of medication" would motivate better adherence, younger asthmatics may feel more healthy and might underestimate their disease severity compared to older patients and more likely be nonadherent to ICS. In a similar setting survey in Japan on inpatients admitted for asthma exacerbation, ${ }^{23}$ similar age-related trend towards increased adherence were found. They also found that comorbid conditions were more frequent in older age-group, as expected. This would explain the high proportion of nonadherent patients with Charlson index-score $=0$ found in our study to be age-related rather than comorbid conditions.

Overuse of SABA has been associated with clinically uncontrolled asthma ${ }^{24}$ and the apparent preference of reliever medication over maintenance therapy among asthmatics ${ }^{11}$ would suggest that high use of SABA might be associated with poor adherence to ICS. Surprisingly, our results showed that the proportion of patient adherent to ICS increased with higher annual use of SABA (Figure 2 ). As frequent $\mathrm{SABA}$ use is directly connected with frequent acute symptoms (i.e. severe disease), ${ }^{25}$ in addition that our results showed better adherence in patients that have used OCS in the year prior to admission. Together, these results might suggest that the increased level of adherence among these patients is possibly due to severity of disease. As better adherence to medication are more often seen in patients with more severe disease and more disease-related morbidity.

Even though very few patients dispensed antipsychotic in our population, poor adherence was found in this study in both patientgroups who had filled antipsychotic or antidepressant. Smith et al., ${ }^{26}$ in a study on inner-city patients hospitalized for asthma exacerbation with defined nonadherence as $<50 \%$ of described medications, found that patients with high levels of depressive symptoms is associated with an increased risk of poor adherence. Several features of depression and psychosocial risk factors may influence adherence including poor motivation (apathy), pessimism over effectiveness of treatment, self-neglect, and intentional self-harm.

There were 20 patients using anticholinergic in our study population, which are used mainly for treatment of chronic obstructive pulmonary disease (COPD), surprisingly none of these patients were non-users of ICS. This might indicate either they were using both medications or a change of indication was made during the period preceding admission. Regardless we could not dismiss the fact that these patients more likely are COPD patients rather than asthmatics, as the addition of FDC of ICS/LABA to maintenance therapy of anticholinergic are recommended by guidelines for patients with severe COPD with frequent exacerbations. ${ }^{27}$ But because a clear discharge diagnosis of asthma as main diagnosis as of the rest of the study population these patients could not be excluded from our results.

This study was limited as there was no record of patients' prescribed daily doses. Thus, we had to use a fixed dose generalized for our whole population to define adherence with ICS. As DDD reflect the assumed average dose $\mathrm{e}^{14}$ in the general population, in our hospitalized setting which comprised mostly of patients with more severe asthma, applying DDD as an indicator for patients' dosing regimen might be understated. In addition, the low dose of which we based our measure of adherence may also be cause to believe that the actual proportion of nonadherence might be greater than what we found.

The main strength in our study was the use of electronic pharmacy records to account for patients medication use. As prescription databases (OPED) are extensive and records completely covers all dispensed drugs avoids for recall bias compared with selfreporting, or in the case of medication measurement where misplaced canisters and patients deliberately dump medication to appear more adherent. Another advantage is by assessing prescription records retrospectively meant that this study were free from the Hawthorne effect, often seen in subjects when under observation tend to improve on their adherence to medication. However, prescription filling only accounts for medication dispensed, and not medication actually used by patients. Consequently, surrogate method of measuring adherence may overestimate adherence in this group.

These findings indicate several factors associated with adherence including increasing age, increased use of SABA, FDC therapy, the use of rescue therapy (i.e. OCS) and the use of antidepressant. Even though our study population comprised mostly of patients with more severe disease, overall use of reliever medication was relatively low, as the majority were in the lowest category of SABA use. The overreliance on as-need reliever medication seems not as extensive among asthma hospitalized patients as first thought. However, this study demonstrates that non adherence still remains a problem among patients hospitalized for asthma exacerbation. A sizable sample was not in treatment with ICS the year preceding admission, although not able to determine whether patients were under diagnosed or it was due to non-filling; regardless suggests that there is still room for improvement. Evidence-based guidelines are clear on recommendation for treatment of asthma; attention now needs to be focused on interventions that enhance implementation.

\section{Acknowledgements}

None. 


\section{Conflict of interest}

The author declares no conflict of interest.

\section{References}

1.http://www.ginasthma.org

2.https://heinz.sdu.dk:8443/ws/files/5004/SUSY.pdf

3. Barnes PJ, WoolcockAJ. Difficult asthma. Eur Respir J. 1998;12(5):12091218 .

4. Williams LK, Pladevall M, Xi H, et al. Relationship between adherence to inhaled corticosteroids and poor outcomes among adults with asthma. J Allergy Clin Immunol. 2004;114(6):1288-1293.

5. Gamble J, Stevenson M, McClean E, et al. The prevalence of nonadherence in difficult asthma. Am J Respir Crit Care Med. 2009;180(9):817822.

6. Suissa S, Ernst P, Kezouh A. Regular use of inhaled corticosteroids and the long term prevention of hospitalisation for asthma. Thorax. 2002;57(10):880-884.

7. Suissa S, Ernst P, Benayoun S, et al. Low-dose inhaled corticosteroids and the prevention of death from asthma. N Engl J Med. 2000;343(5):332336 .

8. Donahue JG, Weiss ST, Livingston JM, et al. Inhaled steroids and the risk of hospitalization for asthma. JAMA. 1977;277(11):887-891.

9. Suissa S, Ernst P. Inhaled corticosteroids: Impact on asthma morbidity and mortality. J Allergy Clin Immunol. 2001;107(6):937-944.

10. Bender B, Milgrom H, Rand C. Nonadherence in asthmatic patients: is there a solution to the problem? Ann Allergy Asthma Immunol. 1997;79(3):177-187.

11. Lindsay JT, Heaney LG. Nonadherence in difficult asthma-facts, myths, and a time to act. Patient Prefer Adherence. 2013;7:329-326.

12. Davidsen JR, Sondergaard J, Hallas J, et al. Increased use of inhaled corticosteroids among young Danish adult asthmatics: An observational study. Respir Med. 2010;104(12):1817-1824.

13. Hallas J. Drug utilization statistics for individual level pharmacy dispensing data. Pharmacoepidemiol Drug Saf. 2005;14(7):455-463.

14. http://www.whocc.no/filearchive/publications/2014_guidelines.pdf
15. http://pro.medicin.dk/Laegemiddelgrupper/Grupper/263058\#a000

16. Charlson ME, Pompei P, Ales KL, et al. A new method of classifying prognostic comorbidity in longitudinal studies:development and validation. J Chronic Dis. 1987;40(5):373-383.

17. Williams LK, Joseph CL, Peterson EL, et al. Patients with asthma who do not fill their inhaled corticosteroids: A study of primary nonadherence. J Allergy Clin Immunol. 2007;120(5):1153-1159.

18. Bronstein JM, Santer L, Johnson V. The use of medicaid claims as a supplementary source of information on quality of asthma care. J Healthc Qual. 2000;22(6):13-18.

19. Watts RW, McLennan G, Bassham I, et al. Do patients with asthma fill their prescriptions? A primary compliance study. Aust Fam Physician. 1997;26(Suppl 1):S4-S6.

20. Aubier M, Pieters WR, Schlosser NJJ, et al. Salmeterol/fluticasone propionate $(50500 \mu \mathrm{g})$ in combination in a Diskus inhaler (Seretide) is effective and safe in the treatment of steroid-dependent asthma. Respir Med. 1999;93(12):876-884.

21. Zetterstrom O, Buhl R, Mellem H, et al. Improved asthma control with budesonide/formoterol in a single inhaler, compared with budesonide alone. Eur Respir J. 2001;18(2):262-268.

22. Pauwels RA, Lofdahl CG, Postma DS, et al. Effect of Inhaled formoterol and budesonide on exacerbations of asthma. $N$ Engl J Med. 1997;337(20):1405-1411.

23. Sekiya K, Taniguchi M, Fukutomi Y, et al. Age-specific characteristics of inpatients with severe asthma exacerbation. Allergol Int. 2013;62(3):331-336.

24. Davidsen JR, Hallas J, Sondergaard J, et al. Association between prescribing patterns of anti-asthmatic drugs and clinically uncontrolled asthma:a cross-sectional study. Pulm Pharmacol Ther. 2011;24(6):647653.

25. Diette GB, Wu AW, Skinner EA, et al. Treatment patterns among adult patients with asthma: factors associated with overuse of inhaled $\beta$-agonists and underuse of inhaled corticosteroids. Arch Intern Med. 1999; 159(22):2697-2704.

26. Smith A, Krishnan JA, Bilderback A, et al. Depressive symptoms and adherence to asthma therapy after hospital discharge. Chest. 2006;130(4):1034-1038.

27. http://pro.medicin.dk/Sygdomme/Sygdom/318294\#a000 